\title{
Wann ist die sog. Chronikerziffer im EBM berechnungsfähig?
}

— Die Leistung nach Nr. 03212 EBM steht für einen „Zuschlag zu den Versichertenpauschalen EBM 03110 bis 03112 bei der Behandlung eines Versicherten mit einer oder mehreren schwerwiegenden chronischen Erkrankung(en) gemäß §2 Absatz 2 der Richtlinie des Gemeinsamen Bundesausschusses zur Definition schwerwiegender chronischer Krankheiten im Sinne des Paragraf 62 SGB V“. Eine Krankheit ist demnach schwerwiegend chronisch, wenn sie wenigstens ein Jahr lang, mindestens einmal pro Quartal ärztlich behandelt wur- de (Dauerbehandlung) und eines der folgenden Merkmale vorhanden ist: Es liegt eine Pflegebedürftigkeit der Pflegestufe 2 oder 3 nach dem zweiten Kapitel SGB XI vor. Es liegt ein Grad der Behinderung (GdB) von mindestens $60 \%$ oder eine Minderung der Erwerbsfähigkeit (MdE) von mindestens $60 \%$ vor, wobei der GdB oder die MdE nach den Maßstäben des $\$ 30$ Abs. 1 BVG oder des $§ 56$ Abs. 2 SGB VII festgestellt und zumindest auch durch die Krankheit nach Satz 1 begründet sein muss. Es ist eine kontinuierliche medizinische Versor-

\section{Ist die elektronische Gesundheitskarte ein „Kuckucksei“ der EU?}

- Nach Auffassung von Gesundheitsexperten in der EU kann das elektronische Gesundheitssystem die Beziehung zwischen Arzt und Patient nicht ersetzen, aber es stecken phantastische Möglichkeiten darin und damit kann es einen wichtigen Beitrag bei der Modernisierung der Gesundheitsfürsorge leisten. Gedacht ist an eine Verbesserung der Qualität der Gesundheitsfürsorge z.B. durch Kostensenkung bei der medizinischen Versorgung, Reduzierung der stationären Verweildauer, im Hinblick auf die Zunahme von chronisch Kranken sowie mit Blick auf die Möglichkeit einer unabhängigeren Lebensführung von Patienten in abgelegenen Gebieten.

Konkret stand dabei der Austausch der Möglichkeiten, die sich über den verstärkten Einsatz der Informations- und Kommunikationstechnologien (IKT) ergeben (z. B. Erstellung von Ferndiagnosen, Fernüberwachung des Gesundheitszustandes von Patienten, elektronische Übermittlung von Patientendaten) im Vordergrund der Beratungen. Im Bereich des Ausbaus dieser Dienste möchte man zunächst die Tele- überwachung für die Kontrolle chronisch Kranker, die an Diabetes und Herzerkrankungen leiden, fördern. Darüber sollen die Mitgliedstaaten in dem freiwilligen Netzwerk "elektronische Gesundheitsdienste" intensiv zusammenzuarbeiten.

\section{MMW Kommentar}

Wer glaubt, die Datenpanne bei der Einführung der elektronischen Gesundheitskarte (eGK) in Deutschland sei zugleich deren Ende gewesen, irrt. Auf europäischer Ebene wird seit über 20 Jahren die Weiterentwicklung der e-Health-Technologien betrieben. Das Ziel ist der Zugang zu einer sicheren, guten Gesundheitsfürsorge überall in der EU. Hier lauert zugleich aber für Deutschland ein ähnlicher Gau wie zurzeit bei den europäischen Regionalhaushalten und deren Defizitausgleich. Kommt es im Rahmen der Einführung der eGK in Deutschland und zugleich auch einer europäischen Gesundheitskarte zu einem Run auf bundesdeutsche Gesundheitsleistungen, könnte das zum finanziellen Kollaps des Systems der gesetzlichen Krankenversicherung in unserem Land führen. gung (ärztliche oder psychotherapeutische Behandlung, Arzneimitteltherapie, Behandlungspflege, Versorgung mit Heilund Hilfsmitteln) erforderlich, ohne die nach ärztlicher Einschätzung eine lebensbedrohliche Verschlimmerung, eine Verminderung der Lebenserwartung oder eine dauerhafte Beeinträchtigung der Lebensqualität durch die aufgrund der Krankheit nach Satz 1 verursachte Gesundheitsstörung zu erwarten ist.

\section{MMW Kommentar}

Die Leistung nach Nr. 03212 EBM kann einmal im Quartal abgerechnet werden, wenn diese Kriterien zutreffen und die Versichertenpauschalen nach den Nrn. 03110 bis 03112 berechnet werden konnten (Ausnahme: diabetologische oder HIV/AIDS-Schwerpunktpraxen). Dies setzt wiederum voraus, dass mindestens zwei Arzt-Patienten-Kontakte im Quartal stattfanden und einer der beiden persönlich erfolgte. Der weitere Kontakt kann hingegen mittelbar gewesen sein und z.B. dem Leistungsinhalt der Nrn. 01430 oder 01435 EBM entsprechen.

Beachtenswert ist dabei, dass insbesondere die Konstellation „,wenigstens ein Jahr lang, mindestens einmal pro Quartal ärztlich behandelt" und "es ist eine kontinuierliche medizinische Versorgung (ärztliche oder psychotherapeutische Behandlung, Arzneimitteltherapie, Behandlungspflege, Versorgung mit Heil- und Hilfsmitteln) erforderlich, ohne die nach ärztlicher Einschätzung eine lebensbedrohliche Verschlimmerung, eine Verminderung der Lebenserwartung oder eine dauerhafte Beeinträchtigung der Lebensqualität durch die aufgrund der Krankheit nach Satz 1 verursachte Gesundheitsstörung zu erwarten ist" auf viele Krankheitsbilder zutrifft, die üblicherweise - insbesondere von Kassenvertretern - nicht als chronische Erkrankungen anerkannt werden. Beispielhaft können hier Diagnosen wie "Struma”, "Allergie", "Varicosis" oder auch "orthostatische Dysregulation" genannt werden. 